\title{
Switching and combining device-aided therapies in advanced Parkinson's disease: a double centre retrospective study
}

Dejan Georgiev MD, $\mathrm{PhD}^{1,2}$, Sentilija Delalić MD ${ }^{1,3}$, Nina Zupančič Križnar MD ${ }^{1}$, Achinoam Socher $\mathrm{MD}^{4,5}$, Tanya Gurevich $\mathrm{MD}^{4,5}$, Maja Trošt, $\mathrm{MD}, \mathrm{PhD}^{1,6}$

${ }^{1}$ Department of Neurology, University Medical Centre Ljubljana, Ljubljana, Slovenia ${ }^{2}$ Faculty of Computer and Information Sciences, University of Ljubljana, Ljubljana, Slovenia

${ }^{3}$ General Hospital Isola, Isola, Slovenia

${ }^{4}$ Movement Disorders Unit, Tel-Aviv Sourasky Medical Centre, Tel Aviv University, Tel Aviv, Israel

${ }^{5}$ Sagol School of Neuroscience, Tel Aviv University, Tel-Aviv, Israel ${ }^{6}$ Faculty of Medicine, University of Ljubljana, Ljubljana, Slovenia

Word count: abstract: 250 words, manuscript 3798.

Corresponding author:

Assist. Prof. Dejan Georgiev, MD, PhD

dejan.georgiev@kclj.si

Department of Neurology, University Medical Centre Ljubljana

Zaloška cesta 2, 1000 Ljubljana

Slovenia+386 70708514 


\begin{abstract}
Background: Continuous subcutaneous apomorphine infusion (CSAI), levodopa-carbidopa intestinal gel infusion (LCIG), and deep brain stimulation of the subthalamic nucleus (STN-DBS) have markedly changed the treatment landscape of advanced Parkinson's disease (aPD). Despite a similar outcome of all device-aided therapies (DATs), some patients switch or combine DATs. The aim of this retrospective study was to explore the frequency and reasons for switching between or combining DATs in two movement disorders centres in Slovenia and Israel.

Methods: We collected and analysed demographic and clinical data from aPD patients who switched between or combined DATs. Motor and non-motor reasons and their frequency for switching/combining were examined, as was the effect of DAT using the Global Improvement subscale of the Clinical Global Impression Scale. Nonparametric tests were used to analyse the data.

Results: Of 505 aPD patients treated with DATs at both centres between January 2009 and June 2021, we identified in total 30 patients (6\%), who either switched DAT (N=24: 7 LCIG-to-STN-DBS, 1 LCIG-to-CSAI, 5 CSAI-to STN-DBS, 8 CSAI-to-LCIG, 1 STN-DBS-to-LCIG, 1 LCIG-to-CSAI-to-STN-DBS, and 1 STN-DBSto-CSAI-to-LCIG) or combined DATs (N=6: 5 STN-DBS+LCIG and 1 STN$D B S+C S A I-t o-S T N-D B S+L C I G)$. In most of these patients, inadequate control of motor symptoms was the main reason for switching or combining DATs, but non-motor reasons (related to the disease and/or DAT) were also identified.

Conclusions: Switching between and combining DATs is uncommon, but in some patients brings substantial clinical improvement and should be considered in those who have either inadequate symptom control on DAT treatment or have developed DAT related complications.
\end{abstract}


Keywords: Advanced Parkinson's disease, Deep Brain Stimulation of the Subthalamic Nucleus, L-dopa/Carbidopa Intestinal Gel Infusion, Continuous Subcutaneous Apomorphine Infusion

\section{Introduction}

Parkinson's disease (PD) is a chronic, neurodegenerative disease characterized by the loss of dopamine-producing cells in the substantia nigra pars compacta ( $\mathrm{SNr}$ [1]. Although L-dopa is the most effective treatment for PD, chronic oral L-dopa therapy often leads to motor and non-motor complications, such as motor and non-motor fluctuations, and dyskinesias, which in turn affect the health-related quality of life (HRQoL) of patients and their caregivers [1]. Strategies to alleviate these symptoms, such as increasing the frequency and adjusting the L-dopa dosage, switching to, or adding other dopaminergic drugs, may trigger, or aggravate dyskinesias in their own merits. Indeed, medication management in patients with advanced PD (aPD) may be challenging and generally, not very efficient [2].

Device-aided therapies (DAT), namely, continuous subcutaneous apomorphine infusion (CSAI), levodopa-carbidopa intestinal gel infusion (LCIG), and deep brain stimulation of the subthalamic nucleus (STN-DBS) have markedly changed the landscape of aPD in the last decades. Although all DATs improve patients' motor and non-motor symptoms and HRQoL, they each have certain unique features [3,4]. Several guidelines and algorithms have been published to help neurologists select the most appropriate DAT for each patient [5]. Although the primary goal of all three DATs is to extend ON time without troublesome dyskinesias and improve patients' HRQoL, each has certain advantages and disadvantages to consider when tailoring the treatment for 
individuals with aPD. Despite the personalized and multidisciplinary approach that encourages patients and caregivers to actively participate in shared decision making about the selection of DAT, some patients may not experience a satisfactory treatment outcome and may require a switch to another DAT or combining DATs [6]. This can be distressing for patients and caregivers as well as healthcare professionals.

The aim of this study was to investigate the frequency and reasons for switching between or adding DATs in aPD patients in two tertiary movement disorders centres with a long experience with the three DATs: The Centre for Movement Disorders at the Department of Neurology, University Medical Centre Ljubljana, Slovenia, and the Movement Disorders Unit, at the Tel-Aviv Sourasky Medical Centre, Israel.

\section{Methods}

We retrospectively analysed aPD patients who switched between or added additional DAT from January 2009 to June 2021. The total number of aPD patients that started any DAT in both centres was 505: 251 in Ljubljana, and 254 in Tel Aviv (Figure 1A). Patients who switched the DAT were categorised in seven subgroups and patients who combined DATs in additional two: Switching DATs: 1. LCIG-to-STN-DBS, 2. LCIG-to-CSAI, 3. CSAI-to-STN-DBS, 4. CSAI-to-LCIG, 5. STN-DBS-to-LCIG, 6. LCIG-to-CSAI-to-STN-DBS, 7. STN-DBS-to-CSAI-to-LCIG, and combining DATs: 8. $S T N-D B S+L C I G$ and 9. STN-DBS+CSAI-to-STN-DBS+LCIG (Suppl. Table 1, Figure 1B). 
Table 1. Differences in age at onset of Parkinson's disease (PD), PD duration at $1^{\text {st }}$ Device Aided Therapy (DAT), age at $1^{\text {st }}$ DAT, duration of $1^{\text {st }}$ DAT, age at $2^{\text {nd }}$ DAT, duration of $2^{\text {nd }}$ DAT to June 2021, Global Improvement (GI) subscale of the Clinical Global Impression scale after $1^{\text {st }}$ and $2^{\text {nd }}$ DAT, frequency $(F)$ of motor and non-motor reasons for switch/combination and $\mathrm{F}$ of patients switching and combining treatments in males vs. females The median values and range (in brackets) are presented. Mann-Whitney $U$ test (or $\chi^{2}$ test for the frequency of switch/combined vs gender) and $p$-values are also presented.

\begin{tabular}{|c|c|c|c|c|c|c|c|c|c|c|c|c|}
\hline & $\begin{array}{r}\text { Age at PD } \\
\text { onset }\end{array}$ & $\begin{array}{r}\text { PD duration } \\
\text { at } 1^{\text {st }} \mathrm{DAT} \\
\end{array}$ & $\begin{array}{r}\text { Age at } 1^{\text {st }} \\
\text { DAT }\end{array}$ & $\begin{array}{r}\text { Duration of } 1^{\text {st }} \\
\text { DAT }\end{array}$ & $\begin{array}{r}\text { Age at } 2^{\text {nd }} \\
\text { DAT }\end{array}$ & $\begin{array}{r}\text { Duration } 2^{\text {nd }} \\
\text { DAT }\end{array}$ & $\begin{array}{r}\mathrm{Gl} \text { after } 1^{\text {st }} \\
\text { DAT }\end{array}$ & $\begin{array}{r}\text { Gl after } 2^{\text {nd }} \\
\text { DAT }\end{array}$ & $\begin{array}{r}\mathrm{F}_{\text {motor }} \\
\text { reasons }\end{array}$ & $\begin{array}{r}F_{\text {non-motor }} \\
\text { reasons } \\
\end{array}$ & $\mathrm{F}_{\text {switch }}$ & Fcombined \\
\hline Females $(\mathrm{N}=12)$ & $48.5(29-69)$ & $11.5(3-20)$ & $63(38-72)$ & $1.75(0.08-11)$ & $66.5(44-76)$ & $3(0.08-8)$ & $1.5(1-5)$ & $2(1-4)$ & $1(0-3)$ & $0.5(0-2)$ & 10 & 2 \\
\hline Males $(\mathrm{N}=18)$ & $50.5(36-66)$ & $10(6-28)$ & $60(44-75)$ & $3(0.5-18)$ & 67 (39-79) & $2.5(0.08-9)$ & $2(1-6)$ & $2(1-4)$ & $1(0-3)$ & $1(0-2)$ & 14 & 4 \\
\hline Mann-Whitney $U$ & 21.5 & 18.5 & 23 & 17.5 & 18.5 & 19 & 23.5 & 15.5 & 22 & 18 & \multicolumn{2}{|c|}{$\chi^{2}=0.139$} \\
\hline$p$-value & 0.746 & 0.474 & 0.897 & 0.398 & 0.473 & 0.511 & 0.945 & 0.249 & 0.746 & 0.202 & \multicolumn{2}{|c|}{0.709} \\
\hline
\end{tabular}


Figure 1. A. Number of patients (y-axis) who received continuous subcutaneous apomorphine infusion (CSAI), levodopa-carbidopa intestinal gel infusion (LCIG) and deep brain stimulation of the subthalamic nucleus (STN-DBS) (x-axis) from both centres. B. Number of patients (x-axis) on specific switches and combinations of DATs (y-axis).

A

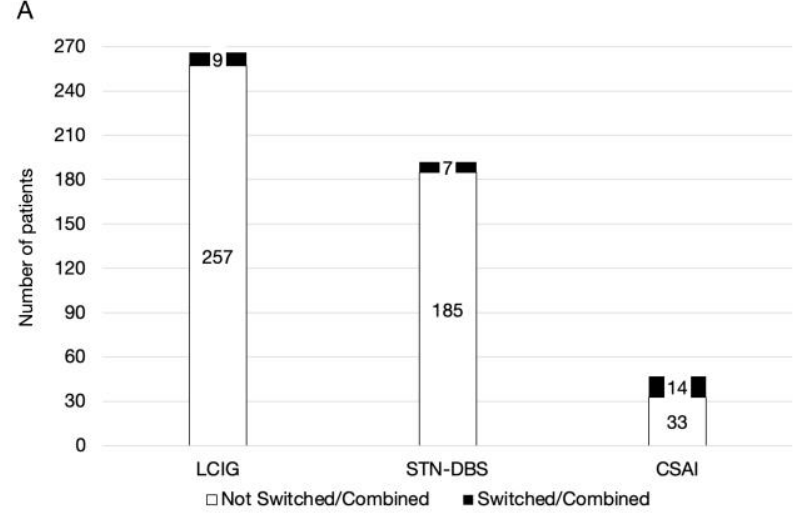

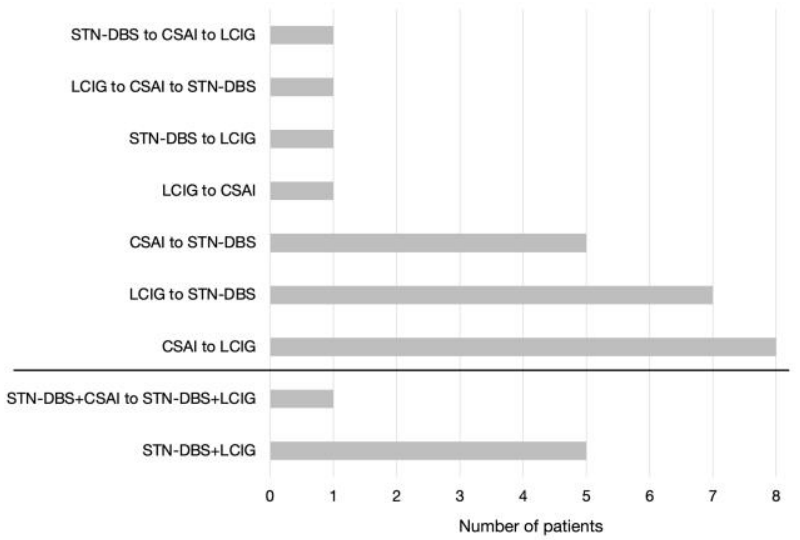

In addition to gender, age at PD onset, PD duration and age at the introduction of 1 st DAT, duration of $1^{\text {st }}$ DAT, age at $2^{\text {nd }}$ DAT, duration of $2^{\text {nd }}$ and $3^{\text {rd }}$ DAT (where applicable) as well as the frequency of motor and non-motor reasons for switching/combining were recorded and analysed. The Global Improvement (GI) subscale from the Clinical Global Impression scale [7] was used to assess the improvement 6 months after introduction of DAT. It assesses the improvement on a scale from 0 to 7 ( $0=$ Not assessed, 1=Very much improved, 2=Much improved, 3=Minimally improved, 4=No change, 5=Minimally worse, $6=$ Much worse, $7=$ Very much worse). For the categories LCIG-to-STN-DBS, CSAI-to-STN-DBS, CSAI-to-LCIG 
and $S T N-D B S+L C I G$, median and range (maximum and minimum) was used as a measure of central tendency. Mann-Whitney $U$ test for two independent samples was used to compare "switch" to "add-on" group regarding the above-mentioned variables. Kruskal-Wallis $H$ test for three or more independent samples was used to compare the following switch categories LCIG-to-STN-DBS, CSAI-to-STN-DBS and CSAI-to-LCIG. For the other categories (LCIG-to-CSAI, STN-DBS-to-LCIG, LCIG-to-CSAI-to-STNDBS, STN-DBS-to-CSAI-to-LCIG and STN-DBS+CSAI-to-STN-DBS+LCIG) containing one or two patients each, the data were presented as absolute values only. Wilcoxon $Z$ test was used for within group analysis of GI after the first and the second DAT. $\chi^{2}$-test was used to compare frequencies $(2 \times 2$ crosstabs $)$ where appropriate. Current state/outcome and reasons for switch/add-on were also recorded and qualitatively analysed. $P$-value of less than 0.05 was considered significant. IBM SPSS for MAC v.26.0 was used for analysis. The study was approved by the Medical Ethical Committee of the Republic of Slovenia.

\section{Results}

The overall number of aPD patients requiring a switch of DAT or additional DAT was 30/505 [(6.0\%), 12 females]. 24/30 aPD patients [(80\%), 10 females] switched DAT, 2 of them switched DAT twice. 6/30 [(20\%), 2 females] patients needed a combined DAT, 1 of them switched between two combined DATs (Figure 1A and B, Suppl. Table 1). There were no significant differences between male and female patients on any of the measured variables (all $p>0.201)$ (Table 1).

Differences between patients switching and combining DATs 
There were no significant differences between patients that switched and combined DATs except for the age at PD onset $(U=30.5, p=0.031$, older in patients with combined DATs), PD duration at first DAT ( $U=29.0, p=0.025$, longer in patients that later switched DAT), duration of first DAT ( $U=27.5, p=0.020$, longer in patients on combined DAT) and frequency of non-motor reasons $(U=33.0, p=0.044$, higher in patients that switched between DATs) (Table 2).

The GI after the first DAT was higher in patients that switched, compared to patients on combined DATs $(U=18.0, p=0.003)$. There was no significant difference in GI between the two groups after the second DAT. In addition, there were no significant differences in GI between first and the second DAT neither in switched nor in the combined group (all $p>0.05$ ).

\section{Differences between subgroups of patients switching DATs}

The three switching categories quantitatively analysed were: LCIG-to-STNDBS, CSAI-to-STN-DBS and CSAI-to-LCIG (Table 3). Age at PD onset, age at $1^{\text {st }}$ DAT and age at $2^{\text {nd }}$ DAT was the highest in CSAI-to-LCIG category, $H(2)=9.03, p=0.011$, $H(2)=8.25, p=0.016$ and $H(2)=7.66, p=0.022$ respectively. PD duration at $1^{\text {st }} \mathrm{DAT}$ was the lowest in the CSAI-to-STN-DBS, $H(2)=9.03, p=0.011$. No significant differences were found for the duration of $1^{\text {st }}$ and $2^{\text {nd }}$ DAT and the frequency of motor and non-motor reasons between categories.

There were no significant differences neither between (all $p>0.309$ ) nor within groups regarding GI after the first and second DAT (all $p>0.317$ ). 
Table 2. Differences in age at onset of Parkinson's disease (PD), PD duration at 1st Device Aided Therapy (DAT), age at $1^{\text {st }}$ DAT, duration of $1{ }^{\text {st }}$ DAT, age at $2^{\text {nd }}$ DAT, duration of $2^{\text {nd }}$ DAT to June 2021, frequency (F) of motor and non-motor reasons for switching or combining therapies, and Global Improvement (GI) subscale of the Clinical Global Impression scale between patients who switched DATs and patients who combined DATs. The median values and range (in brackets) are presented. Mann-Whitney $U$ test, Wilcoxon $Z$ test and $p$-values are also presented. Significant values are written in bold.

\begin{tabular}{|c|c|c|c|c|c|c|c|c|c|c|c|}
\hline & $\begin{array}{r}\text { Age at PD } \\
\text { onset }\end{array}$ & $\begin{array}{r}\text { PD duration } \\
\text { at } 1^{\text {st }} \text { DAT }\end{array}$ & $\begin{array}{r}\text { Age at } 1^{\text {st }} \\
\text { DAT }\end{array}$ & $\begin{array}{r}\text { Duration of } 1^{\text {st }} \\
\text { DAT }\end{array}$ & $\begin{array}{r}\text { Age at } 2^{\text {nd }} \\
\text { DAT }\end{array}$ & $\begin{array}{r}\text { Duration } \\
\text { of } 2^{\text {nd }} D A T \\
\end{array}$ & $\begin{array}{l}\mathrm{F} \text { motor } \\
\text { reasons }\end{array}$ & $\begin{array}{l}\mathrm{F} \text { non-motor } \\
\text { reasons }\end{array}$ & $\begin{array}{l}\text { Gl after } \\
1^{\text {st }} \text { DAT } \\
\end{array}$ & $\begin{array}{l}\text { Gl after } \\
2^{\text {nd }} \mathrm{DAT}\end{array}$ & $\begin{array}{r}\text { Wilcoxon } Z \\
p \text {-value }\end{array}$ \\
\hline Switched $(N=24)$ & $49(29-66)$ & $10(7-28)$ & $60(38-74)$ & $1.75(0.08-18)$ & $66(39-77)$ & $3(0.08-9)$ & $1(0-3)$ & $1(1-3)$ & $2(1-6)$ & $2(1-4)$ & $-0.91, p=0.327$ \\
\hline Combined $(\mathrm{N}=6)$ & $55(46-69)$ & $7(3-11)$ & $62(57-75)$ & $5(3-10)$ & 68 (64-79) & $3(0.08-5)$ & $1(1-2)$ & $0(0-1)$ & $1(1-1)$ & $1.5(1-2)$ & $-1.72, p=0.083$ \\
\hline Mann-Whitney $U$ & 30.5 & 29.0 & 57.5 & 27.5 & 41.5 & 67.5 & 53.0 & 33.0 & 18.0 & 49.5 & 1 \\
\hline$p$-value & 0.031 & 0.025 & 0.452 & 0.020 & 0.113 & 0.813 & 0.347 & 0.044 & 0.003 & 0.251 & / \\
\hline
\end{tabular}

Table 3. Differences in age at onset of Parkinson's disease (PD), PD duration at $1^{\text {st }}$ Device Aided Therapy (DAT), age at $1^{\text {st }}$ DAT, duration of $1^{\text {st }}$ DAT, age at $2^{\text {nd }}$ DAT, duration of $2^{\text {nd }}$ DAT to June 2021, frequency (F) of motor and non-motor causes for switch, and Global Improvement (GI) subscale of the Clinical Global Impression scale after $1^{\text {st }}$ and $2^{\text {nd }}$ DAT between different groups of patients that switched from one to another DAT. Continuous subcutaneous apomorphine infusion (CSAI), Levodopa-carbidopa intestinal gel infusion (LCIG), and Deep brain stimulation of the subthalamic nucleus (STN-DBS). The median values and range (in brackets) are presented. Kruskal Wallis $H$ test, Wilcoxon $\mathrm{Z}$ test and p-values are also presented. Significant values are written in bold.

\begin{tabular}{|c|c|c|c|c|c|c|c|c|c|c|c|}
\hline & $\begin{array}{r}\text { Age at PD } \\
\text { onset }\end{array}$ & $\begin{array}{r}\text { PD duration } \\
\text { at } 1^{\text {st }} \mathrm{DAT} \\
\end{array}$ & $\begin{array}{r}\text { Age at } 1^{\text {st }} \\
\text { DAT }\end{array}$ & $\begin{array}{l}\text { Duration } \\
\text { of } 1^{\text {st }} D A T\end{array}$ & $\begin{array}{r}\text { Age at } 2^{\text {nd }} \\
\text { DAT }\end{array}$ & $\begin{array}{r}\text { Duration of } \\
2^{\text {nd }} D A T\end{array}$ & $\begin{array}{r}F_{\text {motor }} \\
\text { causes }\end{array}$ & $\begin{array}{r}\text { non-motor } \\
\text { causes } \\
\end{array}$ & $\begin{array}{r}\text { Gl after } 1^{\text {st }} \\
\text { DAT }\end{array}$ & $\begin{array}{r}\text { Gl after } 2^{\text {nd }} \\
\text { DAT }\end{array}$ & $\begin{array}{r}\text { Wicloxon } Z \\
p \text {-value } \\
\end{array}$ \\
\hline LCIG-to-STN-DBS (N=7) & $45(29-50)$ & $11(9-28)$ & $59(38-69)$ & $4(1-11)$ & $66(44-73)$ & $3(2-8)$ & $1(0-3)$ & $1(0-2)$ & $3(1-3)$ & $2(1-3)$ & $-0.55, p=0.581$ \\
\hline CSAI-to-STN-DBS $(\mathrm{N}=5)$ & $43(36-52)$ & $8(8-13)$ & $52(44-65)$ & $1(1-2)$ & $54(39-67)$ & $2(1-9)$ & $0(0-2)$ & $1(0-2)$ & $2(1-2)$ & $1(1-3)$ & $-1.00, p=0.317$ \\
\hline CSAI-to-LCIG $(\mathrm{N}=8)$ & $55(48-66)$ & $12(7-20)$ & $70(58-74)$ & $1.5(0.5-5)$ & $72(59-77)$ & $2.75(0.5-5)$ & $1(0-3)$ & $1(0-2)$ & $2(1-3)$ & $2(1-3)$ & $0.00, p=1.000$ \\
\hline Kruskal Wallis $H$ & 9.03 & 9.14 & 8.25 & 3.26 & 7.66 & 0.83 & 1.95 & 0.42 & 0.70 & 2.35 & l \\
\hline$p$-value & 0.011 & 0.010 & 0.016 & 0.196 & 0.022 & 0.662 & 0.377 & 0.809 & 0.705 & 0.309 & l \\
\hline
\end{tabular}




\section{Differences between centres}

The number of patients switching or combing DATs was similar in both centres: $16(6.4 \%$ of 251) from Slovenia and 14 (5.5\% of 254) from Israel. Age at PD onset was higher in patients from TAMSC, $U=64.0, p=0.046$. While disease duration at introduction of $1^{\text {st }}$ DAT was longer in UMCL patients, $U=56.0, p=0.019$, duration of the $1^{\text {st }}$ DAT was longer in TAMSC patients, $U=59.0, p=0.027$. Non-motor reasons for switching and combining were more common in patients from UMCL, $U=17, p=0.001$. All patients with combined DATs were from TAMSC, no combined DATs were noted in patients from UMCL, $\chi^{2}=8.571, p=0.003$ (Table 4). 
Table 4. Differences in frequency of switching and combining ( $F$ switch/Add $)$, age at onset of Parkinson's disease (PD), PD duration at $1^{\text {st }}$ Device Aided Therapy (DAT), age at $1^{\text {st }}$ DAT, duration of $1^{\text {st }}$ DAT, age at $2^{\text {nd }}$ DAT, duration of $2^{\text {nd }}$ DAT to June 2021, Global Improvement (GI) subscale of the Clinical Global Impression scale after $1^{\text {st }}$ and $2^{\text {nd }}$ DAT, frequency $(F)$ of motor and non-motor causes for switch/combination between centres University Medical Centre Ljubljana (UMCL) and Tel-Aviv Sourasky Medical Centre (TASMC). The median values and range (in brackets) are presented. Mann-Whitney U test, and p-values are also presented. Significant differences are presented in bold.

\begin{tabular}{|c|c|c|c|c|c|c|c|c|c|c|c|}
\hline & Gender & $\begin{array}{r}\text { Age at } \\
\text { onset }\end{array}$ & $\begin{array}{r}\text { PD duration } \\
\text { at } 1^{\text {st }} \text { DAT } \\
\end{array}$ & $\begin{array}{r}\text { Age at } 1^{\text {st }} \\
\text { DAT }\end{array}$ & $\begin{array}{r}\text { Duration } \\
\text { of } 1^{\text {st }} \text { DAT }\end{array}$ & $\begin{array}{r}\text { Age at } 2^{\text {nd }} \\
\text { DAT }\end{array}$ & $\begin{array}{r}\text { Duration } 2^{\text {nd }} \\
\text { DAT } \\
\end{array}$ & $\begin{array}{l}\text { Gl after } \\
1^{\text {st }} \text { DAT }\end{array}$ & $\begin{array}{l}\text { Gl after } \\
2^{\text {nd }} D A T\end{array}$ & $\mathrm{~F}_{\text {motor causes }}$ & $\mathrm{F}_{\text {non-motor causes }}$ \\
\hline UMCL $(\mathrm{N}=16)$ & $2(1-2)$ & $48(36-66)$ & $10.5(8-28)$ & $60(44-74)$ & $1(0.08-11)$ & $65.5(39-77)$ & $2.5(0.08-9)$ & $2(1-4)$ & $2(1-4)$ & $1(0-3)$ & $1(0-2)$ \\
\hline TAMSC $(\mathrm{N}=14)$ & $2(1-2)$ & $53(29-69)$ & $8.5(3-15)$ & $62(38-75)$ & $4(0.5-18)$ & 67 (44-79) & $3.25(0.08-5)$ & $1.5(1-6)$ & $2(1-4)$ & $1(1-3)$ & $0(0-1)$ \\
\hline Mann-Whitney U & 106 & 64 & 56 & 102 & 59 & 88.5 & 110 & 97 & 104.5 & 76 & 17 \\
\hline$p$-value & 0.769 & 0.046 & 0.019 & 0.677 & 0.027 & 0.328 & 0.933 & 0.512 & 0.743 & 0.102 & 0.001 \\
\hline
\end{tabular}


Reasons for switching between DATs

LCIG-to-STN-DBS: 7/24 (29.1\%) patients switched. Unsatisfactory control of motor symptoms was the reason for switching in 6/7 patients: persistent dyskinesias in 4, continuous motor fluctuations in 3, and freezing of gait along with tremor in 1 . Additional non-motor causes for switching were found in 5/7 of these patients, in some there were more than one. LCIG-related polyneuropathy, which was the most common non-motor reason for switch to STN-DBS, was observed in 3, followed by weight loss in 2 , punding in 1 , sleep problems in 1 and excessive sweating in 1 patient.

LCIG-to-CSAI: One patient switched due to unsatisfactory control of dyskinesias with LCIG.

CSAI-to-STN-DBS: 5/24 (20.8\%) switched. Motor reasons were noted in 2: OFF related dystonia of respiratory muscles and dyskinesias in 1 patient, and unsatisfactory control of tremor in 1 . One patient reported bothersome daytime sleepiness and nausea still present one year after CSAI introduction. In 1 patient skin nodules at injection sites were the main reason for switching. In 2/5, CSAI was introduced as a "bridging therapy" while waiting for STN-DBS. Although both patients benefited well from CSAI, they preferred to switch to STN-DBS.

CSAI-to-LCIG: 8/24 (33.3\%) patients switched. Motor reasons (fluctuations in 4 , dyskinesias in 2 , freezing of gait in 1 , and OFF dystonia in 1 ) were noted in $7 / 8$. Nonmotor reasons were also noted in 5/8 - psychosis in 1 , visual hallucinations along with sleepiness and fatigue in 1 , subjective cognitive complaints in 1 , skin nodules at the injection sites were noted in 2 patients and skin necrosis in additional 1. In patients in whom motor fluctuations were the reason for change, improvement was observed after switching to LCIG. 
STN-DBS-to-LCIG: One patient switched because of ongoing severe motor fluctuations and dyskinesias. The DBS was switched off, but not removed. This patient deteriorated relentlessly and deceased 1 year later.

Two patients switched DATs twice. One patient first switched from LCIG-toCSAI due to severe dyskinesias and autonomic storms during wearing OFF periods. This same patient shortly later switched from CSAI-to-STN-DBS because neither dyskinesia nor autonomic storms improved on CSAI treatment. Patient's condition improved considerably on STN-DBS and the treatment was efficient for 12 years but then had to be removed due to infection of the DBS system. The other patient switched from STN-DBS-to-CSAI because of OFF related dystonia and cognitive deterioration without any improvement. Two months later the patient was switched to LCIG with partial improvement. Similarly, the DBS was switched off, but not removed.

\section{Reasons for combining DATs}

$5 / 6(83.3 \%)$ patients had a combination of STN-DBS (first)+LCIG (added). In 3 of these patients, only unilateral STN-DBS was inserted due predominantly unilateral presentation of the disease in 2 . In 1 , the unilateral surgery was the first step of planned bilateral procedure due to increased risk for cognitive deterioration in patient with mild cognitive decline and depression, but the second electrode was never inserted. All 3 patients had continuous motor fluctuations despite unilateral DBS. One of the 3 mentioned patients with unilateral surgery suffered from a severe form of dopamine dysregulation syndrome (DDS) and combined LCIG without satisfactory outcome and was one month later switched back to STN-DBS only. The additional 2 patients with combined DAT had bilateral STN-DBS. Freezing of gait was the reason for the addition of LCIG in 1, and motor fluctuations and dysphagia in the other. 
One patient out of six with combined DAT was first on STN-DBS and had CSAI added because of gait impairments and falls after a period of 10 years of successful STN-DBS treatment. The voltage of stimulation was reduced and CSAI introduced with a good disease control for another 5 years. After this period, motor fluctuations became difficult to control with this combination and, instead of CSAI, LCIG was introduced with a favourable control of motor symptoms. Interestingly, urinary retention that appeared when the patient was on $S T N-D B S+C S A I$ resolved on $S T N-D B S+L C I G$. To the best of our knowledge, urinary retention has not been reported as a side effect of CSAI. However, the effect of dopaminergic medication on bladder control is unpredictable and studies to date have yielded conflicting results [8], with some studies showing that L-dopa and apomorphine improve bladder hyperactivity [9], while other studies show an unpredictable effect [10].

\section{Motor vs. non-motor reasons for switching and combining DATs}

Multiple motor and non-motor reasons were identified in most of the patients switching and combining DATs (24/30). Only 6 patients had a single reason for the change of therapy (Suppl. Table 1).

Among motor reasons for switching, persisting motor fluctuations was the most common one for switching (9/24) or combining DATs (5/6) (Figure 2). The second most common cause for switching were dyskinesias $(8 / 24)$, followed by dystonia (5/24), tremor and freezing of gait (2/24 each). Less common causes for double DATs were dyskinesias, dystonia, freezing of gait and gait impairment (1/6 each).

Figure 2. Motor (black bars) and non-motor (grey bars) reasons for switching between or combining device added therapies (DAT) (y-axis). X-axis - number of patients. 


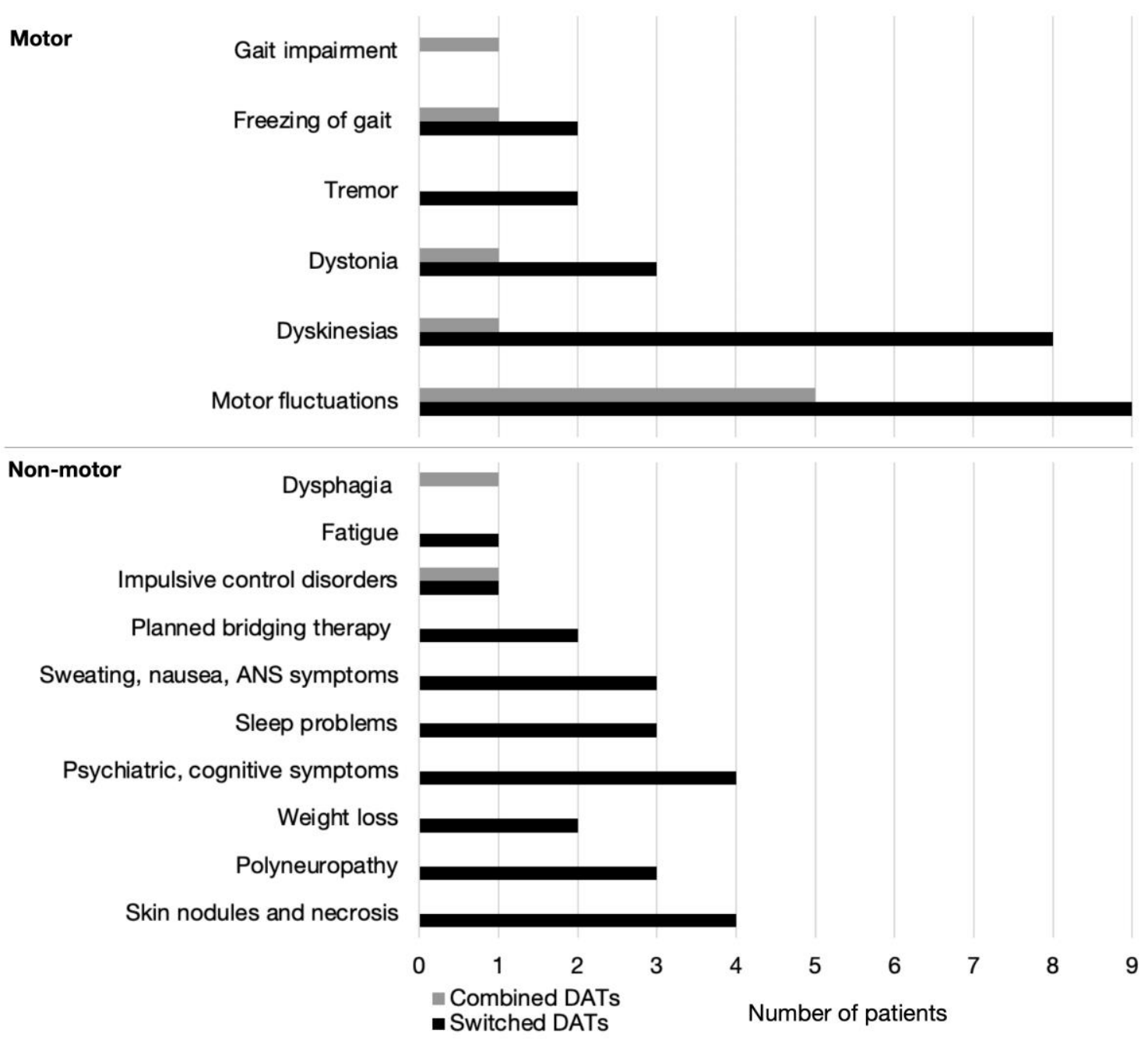

Non-motor causes were much less frequently the reason for switching DATs. These were divided in treatment related: skin nodules in 3 and skin necrosis in 1 patient on CSAI, polyneuropathy in 3 and weight loss in 2 patients on LCIG. Psychiatric and cognitive complains were the reason for switch in 4 patients, sweating, nausea, and other autonomic nervous system problems in 3, sleep problems in 3, punding in 1 and fatigue in another 1. Likewise, in patients on combined DAT only 2 non-motor reasons in 2 patients, were observed: dysphagia in 1 and DDS in another. 


\section{Discussion}

Our results show that the overall rate of switching between DATs or combined DATs is rather low at $6.0 \%$, implying that DATs are efficient in the vast majority of aPD patients. Most of the needed switches were from CSAI to either STN-DBS or LCIG, followed by switches from LCIG to either STN-DBS or CSAI. Only 2 of the patients switched from STN-DBS: 1 to LCIG and another one to CSAI first and then to LCIG. Six patients were identified with combined therapies, all of whom had STNDBS as their first DAT. GI 6 months after initiation of the first and second, switched or added DAT was similar, arguing that switching or adding DAT is warranted in patients in whom the beneficial effect either wanes or is insufficient.

\section{Switching between DATs}

Age at PD onset, at 1st and 2nd DAT, and PD duration at 1st DAT were highest in patients who switched from CSAI to LCIG and lowest in those who switched from CSAI to STN-DBS. Because CSAI is the least invasive, patients often choose it first. The duration of CSAI treatment is known to be short [11], lasting only a few years, and younger patients intuitively prefer to switch to STN-DBS when needed, while for older patients LCIG is the better option.

As expected, there was no difference in GI between firstly introduced DATs, confirming a similar effect of all of them. Motor causes (unsatisfactory control of motor fluctuations and dyskinesias) were the most common reason for switch and similar in all switching combination. This is not entirely consistent with the results of a recent randomized trial which showed that LCIG was effective in reducing dyskinesias [12], however, in our study the percentage of observed aPD patients who required switching was low. Our results are similar to the findings of a recent study in which 19 patients 
were sequentially or in simultaneously treated with STN-DBS and LCIG [13]. Despite the good initial effect of the first DAT, recurrence of motor fluctuations and dyskinesias were the most common reason for switching or combining therapies in this Dutch study. Different reasons might have been responsible for the findings in this study, such as younger age of patients at first DAT, presuming longer disease duration and further development of motor complications, higher expectations from the DAT, and unilateral STN-DBS [13]. In our study, most switches were from CSAI to either LCIG or STNDBS. In a few patients, CSAI was intended as a bridging therapy while patients waited for surgery. These patients had the option to remain on CSAI but preferred to switch. A recent study that directly compared the effect of consecutive treatment with CSAI and STN-DBS [14] showed similar results to ours: STN-DBS had a better overall effect than CSAI, but because CSAI is non-invasive, easy to use and provides a good benefit for motor symptoms, CSAI may be a convenient treatment option for aPD patients waiting STN-DBS.

OFF related respiratory dystonia was another observed and already described [15] very disabling motor reason for switch from CSAI to STN-DBS. As expected, and previously described skin nodules (and necrosis in one patient) were the main nonmotor reason for switching from CSAI to another DAT $[11,16,17]$. In our study, only one patient switched from CSAI to LCIG due to psychotic symptoms. Interestingly, the age of onset as well as age at first and second DAT were the highest in the CSAI-to-LCIG group. Most likely these patients were not suitable candidates for STN-DBS due to their older age at the time of switching. Consistent with this observation, the CSAI-to STNDBS group was the youngest with the shortest PD duration before the introduction of DAT. 
In our study, most switches from LCIG were to STN-DBS. Again, in most cases, the reasons were unsatisfactory control of motor fluctuations and dyskinesias. However, polyneuropathy (in addition to unsatisfactory control of motor symptoms) was the reason for switching from DAT in 3 patients and weight loss in one patient. Both are known side effects of LCIG [18], but may be under-recognised by clinicians in the early years of LCIG treatment. Nowadays, proactive screening for possible polyneuropathy by vitamin B12 blood levels and electromyography is performed in LCIG candidates before and after initiation [19].

Interestingly, 2 patients switched from LCIG-to-CSAI, both because of uncontrollable motor symptoms. However, 1 of them switched further from CSAI to STN-DBS in only a few weeks. Inadequate control of motor fluctuations with very severe dyskinesias and autonomic storm which could not be treated with either of the pump aided treatments improved considerably on STN-DBS in this patient.

One patient switched from STN-DBS-to-LCIG because of recurrence of severe motor fluctuations and dyskinesias after 18 years of successful treatment with STNDBS. The recurrence of motor fluctuations was probably due to overall deterioration in this patient, that deceased one year after the introduction of LCIG with no improvement of his condition despite the switch. The other patient that switched from STN-DBS, first switched to CSAI because of an unsatisfactory control of motor symptoms, in addition to cognitive deterioration. The effect of CSAI was however not sufficient, which lead to the second switch to LCIG with a partial improvement of the symptoms. In both patients the DBS system was switched off, and not removed.

\section{Combining DATs}


Compared to patients who switched to another therapy, these patients were older at PD onset and had a longer duration of the first DAT before the introduction of the second DAT. We did not observe gender differences between patients combining DAT and patients switching to another DAT. Importantly, all the patients who added another DAT were first treated with STN-DBS. Similarly, to the patients who were switching DATs, unsatisfactory control of motor fluctuations and dyskinesias was the main reason for combined DAT in all patients. In 2 of these patients, non-motor symptoms were also identified as an additional cause for double DAT: DDS in 1 and dysphagia in another. Importantly, in 3/5 patients combining STN-DBS with LCIG, unilateral STNDBS was performed, in 1 because of increased risk of surgery and in 2 because of predominantly unilateral presentation of the disease. This is likely the main reason for unsatisfactory control of motor symptoms in these patients. However, in 1 patient unsatisfactory control of motor symptoms after years of bilateral STN-DBS stimulation was a reason for adding LCIG. PD duration at first DAT was shorter in patients combining DATs, and the frequency of non-motor reasons for combining was lower than for switching between DATs. In addition, duration of first DAT was longer in patients with combined DAT. This might be explained by the fact that all patients with combined DAT had STN-DBS as first DAT, which is known for its long-lasting effect on motor and non-motor symptoms of PD [20]. Interestingly, the improvement after the first DAT in these patients was assessed significantly better (lower GI score) than in patients who were switching between DATs (higher GI score), which might explain the decision to combine, rather than to switch DAT.

In only 1 patient CSAI was added to STN-DBS. For 10 years this patient responded very well to STN-DBS, but then developed gait difficulties, falls and dysarthria. The voltage of the stimulation was reduced and CSAI was added with a 
good response for another 5 years. Due to the development of motor fluctuations CSAI was stopped and LCIG introduced in addition to STN-DBS with a beneficial response.

There are several reports of patients receiving combined STN-DBS+LCIG therapy [6,21-23], but only one study prospectively investigated the benefit of adding LCIG to STN-DBS in 19 patients [24]. The main reason for the combined DAT therapy in these patients was prolonged OFF time after the introduction of STN-DBS as well as other symptoms refractory to STN-DBS such as axial symptoms and uncontrollable dyskinesias $[6,21,24]$, due to incorrect patient selection or disease progression requiring high stimulation amplitudes, which can lead to disabling side effects as a result of current spread [6]. In these cases, the addition of LCIG may be regarded as a rescue therapy [23]. In Regidor, Benita, Del Alamo de Pedro, Ley and Martinez Castrillo [24] out of 19 patients treated by STN-DBS+LCIG, 5 patients discontinued combination therapy and returned to DBS monotherapy, 5 discontinued DBS and retained LCIG monotherapy, and 9 patients remained on combination therapy. In 5 patients who discontinued DBS, there was no clinical deterioration after the implantable pulse generator was exhausted, and it was decided not to replace it. In our patients treated with double DAT, motor fluctuations and disruptive dyskinesias decreased after the introduction of LCIG. In addition, axial symptoms, such as freezing of gait, improved too. We also observed improvement in some non-motor symptoms such as DDS and dysphagia.

\section{Limitations of the study}

This study is a retrospective study and therefore the results are prone to bias. However, the data collection and its analysis were done in two different centres using the same methodology. The assessment of GI was also done retrospectively. The quantitative motor assessment of the patients is not presented as these data is not 
available. The main purpose of this study however was to investigate the frequency and causes for switching and combining DATs in PD. The number of patients in the two groups (patients switching DATs and patients with double DATs) was unequal, but the Mann-Whitney $U$ test was used to reliably compare these unequal samples [25]. In addition, there were certain differences between the patients of the two centres, which can be explained by the different structure of switching and combining in the two centres. For example, there were no patients with combined DATs of UMCL and all patients with combined DATs had STN-DBS as their first DAT. Therefore, the differences between the centres are also reflected in the differences between patients switching and combining DATs. The reason for these differences is likely due to local policies regarding the initiation and follow-up of patients with DATs.

\section{Conclusions}

Switching and combining DATs may be a good option for patients with inadequate symptom control or DAT-related complications. Although the indications/criteria for the introduction of all DATs are similar, this should not prevent the introduction or addition of another DAT. We did not demonstrate a valid difference between switching versus dual DAT choice. Most of our patients switched from CSAI to either LCIG or STN-DBS, in a few cases due to planned bridging therapy while waiting for surgery. Close monitoring of DAT effects and side effects is essential. These should be considered in personalized decision making about the most appropriate type and timing of the first, second or combined DAT in each individual patient. 
We thank Ms. Ariela Hilel, nurse in the Movement Disorders Unit, TASMC, for her invaluable help with patient management and data collection, and Ms. Adi Ezra, senior manager in the Movement Disorders Unit, Tel-Aviv Sourasky Medical Centre, Israel for her excellent administrative support.

We would also like to thank all the members of the multidisciplinary team at the Movement Disorders Centre, University Medical Centre Ljubljana, Slovenia, for their compassionate care for advanced PD patients. Special thanks to all patients and their caregivers from both centres for participating in this study.

This research work was supported by the Slovenian Research Agency through the research programme P1-0389, and the research project J7-2600.

Supplementary Table 1. Patients' data

Patients are divided into three groups: Single Switch, Double Switch, and Combined DATs. Medians and range (minimum-maximum) are given for the groups. The treatment-related non-motor reasons for the switch or combined DAT are written in italics. CSAI=continuous subcutaneous apomorphine infusion, DAT=deviceassisted therapy, PD=Parkinson's disease, LCIG=levodopa-carbidopa intestinal gel infusion, STN-DBS =deep brain stimulation of the subthalamic nucleus, TASMC=TelAviv Sourasky Medical Centre, UMCL=University Medical Centre Ljubljana.

\section{References}

1. Lees, A.J.; Hardy, J.; Revesz, T. Parkinson's disease. Lancet 2009, 373, 2055-2066, doi:10.1016/s0140-6736(09)60492-x.

2. Antonini, A.; Moro, E.; Godeiro, C.; Reichmann, H. Medical and surgical management of advanced Parkinson's disease. Movement disorders : official journal of the Movement Disorder Society 2018, 33, 900-908, doi:10.1002/mds.27340.

3. Dafsari, H.S.; Martinez-Martin, P.; Rizos, A.; Trost, M.; Dos Santos Ghilardi, M.G.; Reddy, P.; Sauerbier, A.; Petry-Schmelzer, J.N.; Kramberger, M.; Borgemeester, 
R.W.K.; et al. EuroInf 2: Subthalamic stimulation, apomorphine, and levodopa infusion in Parkinson's disease. Movement disorders : official journal of the Movement Disorder Society 2019, 34, 353-365, doi:10.1002/mds.27626.

4. Martinez-Martin, P.; Reddy, P.; Katzenschlager, R.; Antonini, A.; Todorova, A.; Odin, P.; Henriksen, T.; Martin, A.; Calandrella, D.; Rizos, A.; et al. EuroInf: a multicenter comparative observational study of apomorphine and levodopa infusion in Parkinson's disease. Movement disorders : official journal of the Movement Disorder Society 2015, 30, 510-516, doi:10.1002/mds.26067.

5. Volkmann, J.; Albanese, A.; Antonini, A.; Chaudhuri, K.R.; Clarke, C.E.; de Bie, R.M.; Deuschl, G.; Eggert, K.; Houeto, J.L.; Kulisevsky, J.; et al. Selecting deep brain stimulation or infusion therapies in advanced Parkinson's disease: an evidence-based review. Journal of neurology 2013, 260, 2701-2714, doi:10.1007/s00415-012-6798-6.

6. Kumar, N.; Murgai, A.; Naranian, T.; Jog, M.; Fasano, A. Levodopa-carbidopa intestinal gel therapy after deep brain stimulation. Movement disorders : official journal of the Movement Disorder Society 2018, 33, 334-335, doi:10.1002/mds.27211.

7. Guy, W. ECDEU Assessment Manual for Psychopharmacology; U.S. Department of Health, Education, and Welfare: Rockville, MD, 1976.

8. Winge, K.; Werdelin, L.M.; Nielsen, K.K.; Stimpel, H. Effects of dopaminergic treatment on bladder function in Parkinson's disease. Neurourol Urodyn 2004, 23, 689696, doi:10.1002/nau.20054.

9. Aranda, B.; Cramer, P. Effects of apomorphine and L-dopa on the parkinsonian bladder. Neurourol Urodyn 1993, 12, 203-209, doi:10.1002/nau.1930120302.

10. Fitzmaurice, H.; Fowler, C.J.; Rickards, D.; Kirby, R.S.; Quinn, N.P.; Marsden, C.D.; Milroy, E.J.; Turner-Warwick, R.T. Micturition disturbance in Parkinson's disease. $\mathrm{Br}$ J Urol 1985, 57, 652-656, doi:10.1111/j.1464-410x.1985.tb07025.x.

11. Bhidayasiri, R.; Phokaewvarangkul, O.; Boonpang, K.; Boonmongkol, T.; Thongchuem, Y.; Kantachadvanich, N.; García Ruiz, P.J. Long-term Apomorphine Infusion Users Versus Short-term Users: An International Dual-center Analysis of the Reasons for Discontinuing Therapy. Clin Neuropharmacol 2019, 42, 172-178, doi:10.1097/wnf.0000000000000361.

12. Freire-Alvarez, E.; Kurča, E.; Lopez Manzanares, L.; Pekkonen, E.; Spanaki, C.; Vanni, P.; Liu, Y.; Sánchez-Soliño, O.; Barbato, L.M. Levodopa-Carbidopa Intestinal Gel Reduces Dyskinesia in Parkinson's Disease in a Randomized Trial. Movement disorders : official journal of the Movement Disorder Society 2021, doi:10.1002/mds.28703.

13. van Poppelen, D.; Tromp, A.N.M.; de Bie, R.M.A.; Dijk, J.M. Combined and Sequential Treatment with Deep Brain Stimulation and Continuous Intrajejunal Levodopa Infusion for Parkinson's Disease. $J$ Pers Med 2021, 11, doi:10.3390/jpm11060547.

14. Fernandez-Pajarin, G.; Sesar, A.; Ares, B.; Jimenez-Martin, I.; Gelabert, M.; AranEchabe, E.; Relova, J.L.; Castro, A. Continuous Subcutaneous Apomorphine Infusion before Subthalamic Deep Brain Stimulation: A Prospective, Comparative Study in 20 Patients. Mov Disord Clin Pract 2021, 8, 1216-1224, doi:10.1002/mdc3.13338. 
15. Sage, J.I.; McHale, D.M.; Sonsalla, P.; Vitagliano, D.; Heikkila, R.E. Continuous levodopa infusions to treat complex dystonia in Parkinson's disease. Neurology 1989, 39, 888-891, doi:10.1212/wnl.39.7.888.

16. García Ruiz, P.J.; Sesar Ignacio, A.; Ares Pensado, B.; Castro García, A.; Alonso Frech, F.; Alvarez López, M.; Arbelo González, J.; Baiges Octavio, J.; Burguera Hernández, J.A.; Calopa Garriga, M.; et al. Efficacy of long-term continuous subcutaneous apomorphine infusion in advanced Parkinson's disease with motor fluctuations: a multicenter study. Movement disorders : official journal of the Movement Disorder Society 2008, 23, 1130-1136, doi:10.1002/mds.22063.

17. Sesar, Á.; Fernández-Pajarín, G.; Ares, B.; Rivas, M.T.; Castro, A. Continuous subcutaneous apomorphine infusion in advanced Parkinson's disease: 10-year experience with 230 patients. Journal of neurology 2017, 264, 946-954, doi:10.1007/s00415-017-8477-0.

18. Sensi, M.; Cossu, G.; Mancini, F.; Pilleri, M.; Zibetti, M.; Modugno, N.; Quatrale, R.; Tamma, F.; Antonini, A. Which patients discontinue? Issues on Levodopa/carbidopa intestinal gel treatment: Italian multicentre survey of 905 patients with long-term follow-up. Parkinsonism \& related disorders 2017, 38, 90-92, doi:10.1016/j.parkreldis.2017.02.020.

19. Jick, S.S.; Oleske, D.M.; Persson, R.; Zamudio, J.; Facheris, M.F. Epidemiology of skin event rates among users of pumps for the subcutaneous administration of drugs for chronic conditions. Curr Med Res Opin 2021, 1-9, doi:10.1080/03007995.2021.1953971.

20. Limousin, P.; Foltynie, T. Long-term outcomes of deep brain stimulation in Parkinson disease. Nat Rev Neurol 2019, 15, 234-242, doi:10.1038/s41582-019-0145-9.

21. Klostermann, F.; Jugel, C.; Marzinzik, F. Jejunal levodopa infusion in long-term DBS patients with Parkinson's disease. Movement disorders : official journal of the Movement Disorder Society 2011, 26, 2298-2299, doi:10.1002/mds.23833.

22. Boura, I.; Haliasos, N.; Giannopoulou Iota, A.; Karabetsos, D.; Spanaki, C. Combining Device-Aided Therapies in Parkinson's Disease: A Case Series and a Literature Review. Mov Disord Clin Pract 2021, 8, 750-757, doi:10.1002/mdc3.13228.

23. Elkouzi, A.; Ramirez-Zamora, A.; Zeilman, P.; Barabas, M.; Eisinger, R.S.; Malaty, I.A.; Okun, M.S.; Almeida, L. Rescue levodopa-carbidopa intestinal gel (LCIG) therapy in Parkinson's disease patients with suboptimal response to deep brain stimulation. Ann Clin Transl Neurol 2019, 6, 1989-1995, doi:10.1002/acn3.50889.

24. Regidor, I.; Benita, V.; Del Alamo de Pedro, M.; Ley, L.; Martinez Castrillo, J.C. Duodenal Levodopa Infusion for Long-Term Deep Brain Stimulation-Refractory Symptoms in Advanced Parkinson Disease. Clin Neuropharmacol 2017, 40, 103-107, doi:10.1097/WNF.0000000000000216.

25. Mann, H.B.; Whitney, D.R. On a Test of Whether one of Two Random Variables is Stochastically Larger than the Other. The Annals of Mathematical Statistics 1947, 18, 50-60, 11. 